\title{
Statistical Properties and Applications of Correlated Length Intervals of Some Stationary Random Point Processes
}

\author{
Cherif Bendjaballah \\ Laboratoire des Signaux et Systèmes, CNRS and École Supérieure d' Électricité, 3 rue Joliot-Curie, 91192 Gif-sur-Yvette, France \\ Correspondence should be addressed to Cherif Bendjaballah, bendjaballah@lss.supelec.fr
}

Received 15 November 2011; Accepted 18 December 2011

Academic Editors: Y. Cai and J. Li

Copyright (c) 2012 Cherif Bendjaballah. This is an open access article distributed under the Creative Commons Attribution License, which permits unrestricted use, distribution, and reproduction in any medium, provided the original work is properly cited.

\begin{abstract}
The point process, a sequence of random univariate random variables derived from correlated bivariate random variables as modeled by Arnold and Strauss, has been examined. Statistical properties of the time intervals between the points as well as the probability distributions of the number of points registered in a finite interval have been analyzed specifically in function of the coefficient of correlation. The results have been applied to binary detection and to the transmission of information. Both the probability of error and the cut-off rate have been bounded. Several simulations have been generated to illustrate the theoretical results.
\end{abstract}

\section{Introduction}

It is known that the detection of an optical field at a low level of power is a sequence of events which is a set of distinct time instants $\left\{\vartheta_{j}\right\}, 0 \leq j<\infty$, such that $\vartheta_{j+1} \geq$ $\vartheta_{j}$, for all $j$. These $\left\{\vartheta_{j}\right\} s$, which are the time instants of interaction between the photons and the detector device, for example, a photomultiplier, constitute a random point process (RPP) for which a positive instantaneous density $\lambda\left(\vartheta_{j}\right)$ can be defined. We here consider only simple and homogeneous stationary processes.

Reciprocally, the existence of a physical optical field, given the knowledge of all of the properties of such an RPP, is not an easy problem to solve. For example, given an RPP of nonclassical properties [1] does not necessarily correspond to a nonclassical physical optical field, although its feasibility can be demonstrated [2]. This problem, mainly due to the quantum nature of the interaction between radiation and matter is not examined here.

Nevertheless, using a parameterized RPP, whose special values of such a parameter (denoted here $c$ ) correspond to a physical field, for example, a coherent or a thermal state, then it is reasonable to admit that intermediate values of $c$ corresponds to realistic optical fields. Despite these limitations, the properties of the RPPs studied here are important in statistical optics.
As already pointed out, there are two types of processing that can be utilized to characterize such RPP: the time interval distributions (TIDs) and the probability of number distributions (PNDs) [3]. If we choose to characterize the RPP by the TID, we can define the time interval between points $\theta_{j}=\vartheta_{j+1}-\vartheta_{j}$, called residual time (or lifetime sometimes). Its probability distribution function (PDF) $w(\theta)$ will be called here a triggered PDF. When $\vartheta_{j}=t_{0}$ is arbitrary, that is to say not a point of the (RPP), the $v(\theta)$, PDF of the corresponding $\theta_{j}$ will be called a relaxed PDF.

If we now choose to characterize the RPP by the PND, we can as above, define the relaxed PND $P(n, t)$ of the random variable (RV) $N\left(t_{0}, t_{0}+t\right)$ which is the number of instants occurring within the time interval $\left[t_{0}, t_{0}+\right.$ $t], t_{0}$ and $t \neq \vartheta_{j}$, for all $j$. As previously we may as well define $Q(n, t)$ a triggered PND of the RV $N\left(\vartheta_{j}, \vartheta_{j}+t\right), t \neq \vartheta_{j}$, the counting process being started by a point of the RPP.

The purpose of this paper is firstly to calculate the statistical properties of the RPPs, both in terms of the TIDs and the PNDs. Secondly we apply the results to the calculation of the performances, namely, the probability of error in binary detection and the cut-off rate in the binary transmission of the information, and their variations with respect to the coefficient of correlation $c$. The one-dimensional processes, such as the Poisson and the geometric PNDs, are finally utilized for establishing bounds to the performances. 
In Section 2, the notations are defined and the basic equations are briefly recalled. Section 3 establishes the main results of the PDFs both those dealing with TIDs and PNDs depending on extreme values of $c$ : low values and high values. Finally, Section 4 presents theoretical results and curves that explain and illustrate the results of the numerical simulation.

\section{Basic Equations}

We need to define the Laplace transform of $f(t)$

$$
\rho(s)=\mathcal{L} f(t) \triangleq \int_{0}^{\infty} \mathrm{e}^{-s t} f(t) \mathrm{d} t
$$

and the functions

$$
\begin{aligned}
& \rho_{1}(s)=\frac{\rho(s)}{s(1-\rho(s))}, \\
& \rho_{2}(s)=\frac{\rho(s)(1+\rho(s))}{s(1-\rho(s))} .
\end{aligned}
$$

The PDF of the number of events registered between 0 and $t$, denoted by PND, reads

$$
P(n ; t)=\operatorname{Prob}\left(t \in T=\left[t_{n}, t_{n+1}\right]\right)=\mathrm{E}\left[\ell\left(t_{n}<t<t_{n+1}\right)\right],
$$

where $\ell$ is the indicator function $\ell(t \in T)=1(0)$, in case the event occurs or not within $t \in T$. Therefore

$$
P(n ; t)=\mathcal{L}^{-1} P(n ; s)=\mathcal{L}^{-1}\left(\frac{1-\rho(s)}{s} \rho(s)^{n}\right),
$$

where the symbol $\mathcal{L}^{-1}$ denotes the inverse Laplace transform. From (4), it is easily seen that (see e.g., $[4,5]$ )

$$
\begin{gathered}
\mathrm{E}[N](t)=\mathcal{L}^{-1} \rho_{1}(s), \\
\mathrm{E}\left[N^{2}\right](t)=\mathcal{L}^{-1} \rho_{2}(s) .
\end{gathered}
$$

The symbol $\mathrm{E}$ denotes the mathematical expectation. In the following $P(n ; t)$ will be called the relaxed PDF. Another interesting PDF that can be derived from (4),

$$
Q(n ; t)=\frac{n+1}{\mathrm{E}[N]} P(n+1 ; t),
$$

will be called the triggered PDF. In fact, it is known $[3,6]$ that these PDFs can be expressed using the moments of the time-integrated density

$$
\mathscr{g}(t)=\int_{t_{0}}^{t_{0}+t} \lambda(\vartheta) \mathrm{d} \vartheta
$$

$\mathscr{g}(t)=\lambda t$ for the Poisson RPP. Therefore

$$
\begin{gathered}
P(n ; t)=\frac{1}{n !} \mathrm{E}\left[\mathcal{g}\left(t_{0}+t\right)^{n} \mathrm{e}^{-g\left(t_{0}+t\right)}\right], \\
Q(n ; t)=\frac{1}{\mathrm{E}[N]} \frac{1}{n !} \mathrm{E}\left[g\left(t_{0}+t\right)^{n+1} \mathrm{e}^{-g\left(t_{0}+t\right)}\right] .
\end{gathered}
$$

For all RPPs that we deal with here, all the PDFs depend only on $t$ (not on $t_{0}$ ) due to the stationary property. When $t$ is a parameter, $P(n ; t)$ and $Q(n ; t)$ are simply denoted $P(n)$ and $Q(n)$.

In terms of TIDs, we just recall the basic formulas of $w(t)$ and $v(t)($ see $[6])$

$$
\begin{gathered}
w(t)=\frac{1}{\mathrm{E}[\mathcal{g}]} \mathrm{E}\left[\mathcal{g}\left(t_{0}\right) \mathcal{g}\left(t_{0}+t\right) \mathrm{e}^{-\mathscr{g}\left(t_{0}+t\right)}\right], \\
v(t)=\mathrm{E}\left[\mathcal{g}\left(t_{0}+t\right) \mathrm{e}^{-\mathcal{g}\left(t_{0}+t\right)}\right] .
\end{gathered}
$$

Notice that $g(0) \triangleq w(t=0) / v(t=0)$ the ratio of the values at $t=0$ is related to $\sigma_{g}^{2}$, the variance of $g$, such that $g(0)-$ $1=\sigma_{g}^{2} / \mathrm{E}[\mathscr{g}]^{2}$, where $\sigma_{\mathscr{g}}^{2}$ is the variance of the time-integrated density.

\section{Model for Correlated Variables}

Among the several models proposed to deal with correlated variables, we consider the Arnold-Strauss model $[7,8]$

$$
f(t, u)=K \mathrm{e}^{-(a t+b u+c t u)},
$$

where $a, b, c>0$. The time intervals $t, u$ are of course positive RVs in $[0, \infty[$. The constant of normalization is

$$
K=\frac{c \mathrm{e}^{-a b / c}}{\operatorname{Ei}(1, a b / c)} \longrightarrow \begin{cases}K_{\ell}=a b+c-\frac{c^{2}}{a b} & \text { for } c \ll 1, \\ K_{h}=\frac{c}{1-\gamma-\log (a b)} & \text { for } c \gg 1,\end{cases}
$$

where $\operatorname{Ei}(m, x)$ are the exponential integral functions [9] for $m=1,2, \ldots$, and $x \in \mathbb{R}^{+}$. They verify the approximate expressions given in (A.1a)-(A.2a) in Appendix A.1. The constant $\gamma=0.5772157$ is the Euler constant.

The marginal distribution of $t$ is deduced from (10) and is given by

$$
f(t)=\int_{0}^{\infty} f(t, u) \mathrm{d} u=K \frac{\mathrm{e}^{-a t}}{b+c t} .
$$

In the following, we report some calculations that can be obtained under closed forms depending on the value of the parameter $c$. Let us first consider the case where $c \ll 1$.

3.1. $c \ll 1$. In what follows, most of the calculations are done up to $O\left(c^{3}\right)$.

3.1.1. TID. It can be shown that (see, e.g., [6])

$$
\begin{gathered}
w(t)=K \frac{\mathrm{e}^{-a t}}{b+c t}, \\
v(t) \triangleq \lambda \int_{t}^{\infty} w(\theta) \mathrm{d} \theta .
\end{gathered}
$$

Given $\mathrm{E}[t]$, the first moment of $t$, the average value of the density of the process $\lambda=1 / E[t]$ is given by

$$
\lambda \simeq a+\frac{c}{b}-2 \frac{c^{2}}{a b^{2}} .
$$


Equation (13) yields $\nu(0)=\lambda$ and $\mathrm{d} \nu / \mathrm{d} t=-\lambda w(t)$ which prove that $\nu(t)$ is a monotonic continuously decreasing function of $t$. More precisely, we can show that

$$
\begin{gathered}
w(t) \simeq a^{3} \frac{b^{2}-b c t+c^{2} t^{2}}{a^{2} b^{2}-a b c+2 c^{2}} \mathrm{e}^{-a t}, \\
\nu(t) \simeq a \frac{a^{2} b^{2}-a b c+2 c^{2}-a c(a b-2 c) t+a^{2} c^{2} t^{2}}{a^{2} b^{2}-2 a b c+6 c^{2}} \mathrm{e}^{-a t} .
\end{gathered}
$$

From (15), we deduce

$$
\sigma_{t}^{2} \simeq \frac{1}{a^{2}}\left(1-\frac{2 c}{a b}+\frac{9 c^{2}}{a^{2} b^{2}}\right) .
$$

On the other hand, we can see that

$$
h_{t}(0, c \ll 1)=w(0)-v(0)=\frac{K_{\ell}}{b}-\lambda \simeq \frac{c^{2}}{a b^{2}},
$$

which positivity is a characteristic of classical processes [10].

3.1.2. PND. For a simple approximation, at a first order of $c$, we may use the following:

$$
\begin{aligned}
f(t) \propto & \frac{1}{a b} a \mathrm{e}^{-a t}-\frac{c}{a^{2} b^{2}} a^{2} t \mathrm{e}^{-a t}, \\
P(n, t) \propto & \frac{(a t)^{n}}{n !} \mathrm{e}^{-a t}-\frac{c}{a^{2} b^{2}} \\
& \times\left(\frac{(a t)^{2 n}}{(2 n) !}+\frac{1}{2}\left(\frac{(a t)^{2 n+1}}{(2 n+1) !}+\frac{(a t)^{2 n-1}}{(2 n-1) !}\right)\right) \mathrm{e}^{-a t},
\end{aligned}
$$

where $P(0, t)=(1+a t / 2) \mathrm{e}^{-a t}$ (see [1] pages 789-792).

When only a few values of $P(n, t)$ or only the moments are needed, it is however, better, to use (3) and (5). Based on their expansion as series in $s$, we obtain, at the second order of $c$,

$$
\begin{gathered}
\mathrm{E}[N] \simeq \frac{a^{2}}{a-\omega} t+\frac{a^{2}}{2(a-\omega)^{2}} \omega^{2} t^{2}, \\
\mathrm{E}\left[N^{2}\right] \simeq \frac{a^{2}}{a-\omega} t+\frac{a^{2}\left(2 a^{2}+\omega^{2}\right)}{(a-\omega)^{2}} \omega^{2} t^{2}, \\
\sigma_{n}^{2} \simeq a+\omega+\left(\frac{1}{2}+\frac{1}{a}-a\right) \omega^{2}, \quad(t=1),
\end{gathered}
$$

where we denote $\omega=c / b$.

Seeking the exact expressions of the PDFs of the number seems difficult to obtain. However, as just seen, approximations of closed expressions are simple. Thus, using (4), it is easy to calculate approximate expressions of the PDFs of the number for $n=0,1,2$ and $t=1$, as given in (A.3) in Appendix A.2. Therefore, we can prove that

$$
\begin{aligned}
h_{n}(0, c \ll 1) & =P(0)-P(1) \simeq \mathrm{e}^{-a}\left(1-a-\frac{2-a}{b}\right) c, \\
& =-\frac{1}{e} c-\frac{2}{3 e} c^{2},
\end{aligned}
$$

which is negative for all $c \ll 1$. We will numerically illustrate this property by simulations. When we choose the triggered processing, we obtain

$$
k_{n}(0, c \ll 1)=Q(0)-Q(1) \simeq-\frac{1}{e} c-\frac{1}{4 e} c^{2},
$$

which is here again negative for all $c$.

The case $c \gg 1$ (but finite) is perhaps more interesting although closed forms of the moments are difficult to obtain.

3.2. $c \gg 1$. The calculations are now done up to $O\left(1 / c^{3}\right)$.

3.2.1. TID. We can show that the normalized $w(t)$ is given by $w(t) \sim \frac{c \mathrm{e}^{-a b / c}}{\operatorname{Ei}(1, a b / c)} \frac{\mathrm{e}^{-a t}}{c t+b} \underset{t \neq 0}{\longrightarrow} \frac{\mathrm{e}^{-a t}}{t(\chi-\log c)}\left(1-\frac{b}{c}\left(a+\frac{1}{t}\right)\right)$,

and the unnormalized PDF, evaluated up to $O\left(1 / c^{2}\right)$ and $O(t)$,

$$
\begin{aligned}
& v(t) \sim \lambda \frac{\operatorname{Ei}(1, a(t+b / c))}{\operatorname{Ei}(1, a+b / c)}, \\
& \underset{0<t \ll 1}{\longrightarrow} \lambda \frac{\operatorname{Ei}(1, a t)}{\log (c / a b)-\gamma}-\lambda \frac{b}{c} \frac{1}{\log (a b / c)+\gamma} \frac{\mathrm{e}^{a t}}{t} \\
&-\lambda \frac{a b}{c} \frac{\operatorname{Ei}(1, a t)}{(\log (a b / c)+\gamma)^{2}}, \\
& \sim-\lambda \frac{\gamma+\log (a t)}{\log (a b / c)-\gamma},
\end{aligned}
$$

leading to

$$
h_{t}(0, c \gg 1)=w(0)-v(0)=\frac{K_{h}}{b}-\lambda \simeq \frac{c}{b(1-\chi)}-a \log c,
$$

where we denote $\chi=\gamma+\log (a b)$ and assume that $\lambda \simeq$ $a(\log c-\chi)$. It is seen that $h_{t}(0, c \gg 1)$ given by $(25)$ is now positive and increases more slowly than $h_{t}(0, c \ll 1) \sim c^{2}$ given by (18). From (23), we can also deduce that

$$
\begin{gathered}
\mathrm{E}[t] \sim \frac{1}{a} \frac{1}{\log c-\chi}, \\
\sigma_{t}^{2} \sim \frac{1}{a^{2}} \frac{1}{\log c-\chi}=\frac{\mathrm{E}[t]}{a} .
\end{gathered}
$$

Both moments tend to 0 when $c \rightarrow \infty$.

3.2.2. PND. The approximate expressions given in (A.4) in Appendix A.2 lead to

$$
h_{n}(0, c \gg 1)=P(0)-P(1) \simeq 1+\gamma-\frac{\log c}{c} .
$$

We can calculate the first two moments of the number for the specific case $a=1$ and $b=1$,

$$
\begin{gathered}
\mathrm{E}[N] \propto \log (c), \\
\sigma_{n}^{2} \propto \mathrm{E}[N]^{2} .
\end{gathered}
$$




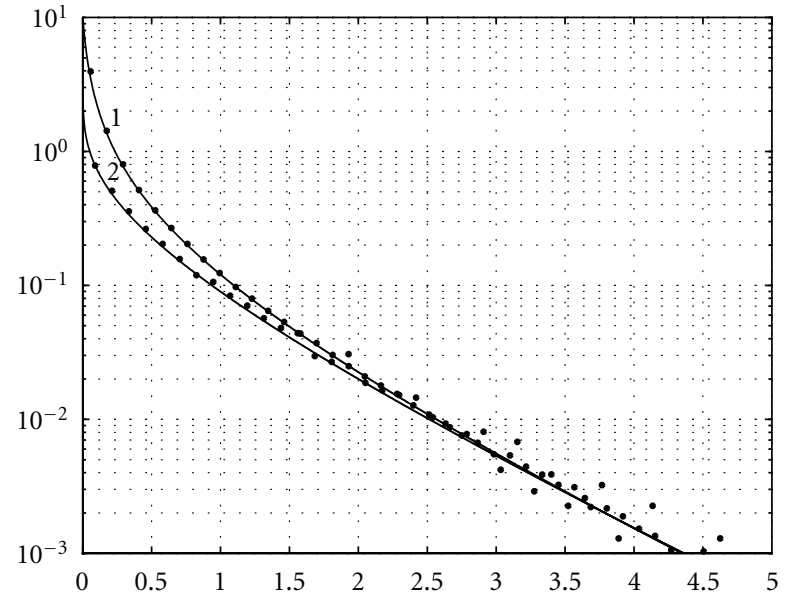

Figure 1: Plots of the Time Interval Distributions $w(t)$ (curve 1) and $v(t)$ (curve 2) versus $t$. The parameters are chosen equal to $a=$ $1, b=1$, where the value of $c=30$ is moderate. The points are the simulated results and the theoretical curves given by (23) and (29) are plotted in solid lines.

As for the previous case, it can be shown that $k_{n}(c) \leq$ 0 , for all $c$.

\section{Simulation and Results}

We have used the algorithm recently described [11] for several values of $c$.

In Figure 1, results of the simulated data of the TIDs are plotted. The $w(t)$, the triggered PDF follows very well (23). The $v(t)$, the relaxed one has been well fitted by the expression

$$
v(t)=\nu_{0} \lambda \frac{\operatorname{Ei}(1, t)}{\operatorname{Ei}(1,1+1 / c)} \underset{0<t \ll 1}{\longrightarrow} \nu_{1} \lambda \frac{\log t}{\log (1 / c)},
$$

for $a=1, b=1, c=30$ and where $\nu_{0}=0.07$ and $\nu_{1}=0.2$.

By the way, it is interesting to remark that the PDF of $S_{\ell}=\sum_{j=0}^{\ell} \theta_{j}, \ell \gg 1$, which is the addition of several correlated identically distributed positive random time interval, deviates from the Gaussian profile. We may conclude that the application of the central limit theorem requires the addition of a very high number of correlated random variables.

On the other hand, for high values of $c$, the theoretical approximations of the PNDs are given by

$$
P(n)=\frac{1}{\mathcal{N}}\left(b_{1} \mathrm{e}^{-a_{1} n}+b_{2} \mathrm{e}^{-a_{2}} \frac{a_{2}^{n}}{n !}\right),
$$

where

$$
\begin{gathered}
\mathcal{N}=b_{2}+\frac{b_{1}}{1-\mathrm{e}^{-a_{1}}}, \\
\mathrm{E}[N]=\frac{1}{\mathcal{N}}\left(a_{2} b_{2}+\frac{b_{1} \mathrm{e}^{a_{1}}}{\left(\mathrm{e}^{a_{1}}-1\right)^{2}}\right),
\end{gathered}
$$

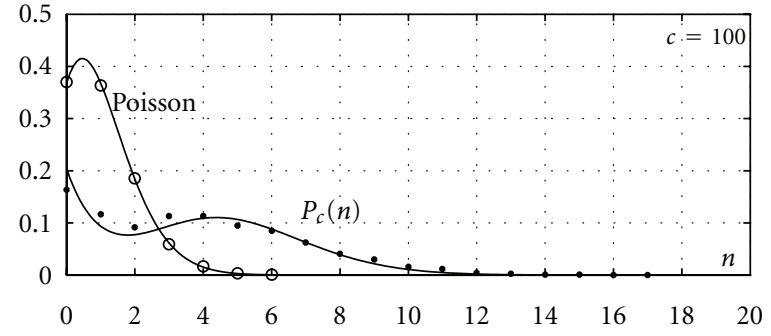

(a)

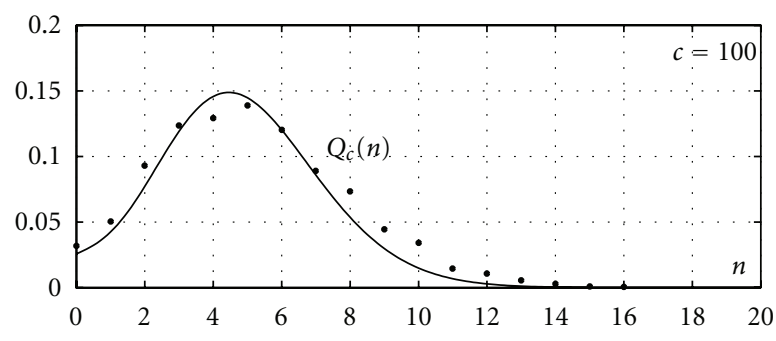

(b)

Figure 2: Plots of the counting PNDs $P(n ; t)$ and $Q(n ; t)$ versus $n$ for the parameters chosen equal to $a=1, b=1, c=100$ and $t=1$. The points are the simulated results and the theoretical approximations given by (30)-(32) are plotted in solid lines. Only integer values of $n$ are meaning. For $c=0$, the PDFs are identical $P(n)=Q(n)$ and are of Poisson type which is drawn in solid line (curve at the top).

leading to

$$
Q(n)=\frac{n+1}{\mathrm{E}[N]} P(n+1) .
$$

The values of the parameters that correctly fit the simulated results of the PDFs, as seen in Figure 2, are $b_{1}=0.2, a_{1}=$ $1, b_{2}=0.62, a_{2}=5.2$, and $\mathrm{E}[N]=3.64$.

The variations of the moments with respect to $c$ are derived from the expressions recalled in the Section 2. We obtained

$$
\begin{gathered}
\mathrm{E}[N] \simeq 1+\gamma+\frac{1}{2} \log c, \\
\sigma_{n}^{2} \simeq 1+\gamma+\frac{1}{4}(1+\log c)^{2} .
\end{gathered}
$$

Regarding the reduced moments $h_{n}$ and $k_{n}$, an excellent fit of the simulated results of Figure 3 is carried out with $\alpha_{1}=$ $4, \beta_{1}=0.17$ and $\alpha_{2}=3, \beta_{1}=0.24$

$$
\begin{aligned}
& h_{n} \simeq \frac{1-\gamma}{2}+\frac{1}{2} \frac{\gamma-\log \left(\alpha_{1} c\right)}{\alpha_{1} c}-\beta_{1}, \\
& k_{n} \simeq \frac{1-\gamma}{2}+\frac{1}{2} \frac{\gamma-\log \left(\alpha_{2} c\right)}{\alpha_{2} c}-\beta_{2},
\end{aligned}
$$

leading to $h_{n} \simeq c^{2} / 2 \log c$ and $k_{n} \simeq-c^{4} / 24(\log c)^{3}$ which are only in qualitative agreement. 


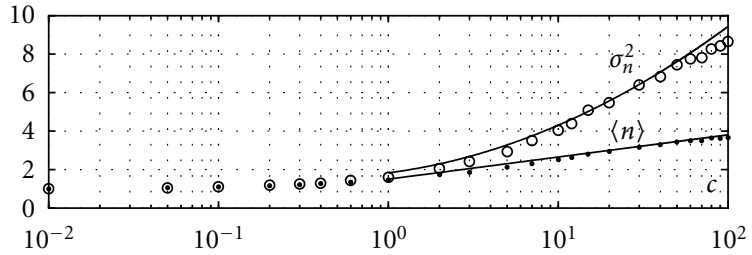

(a)

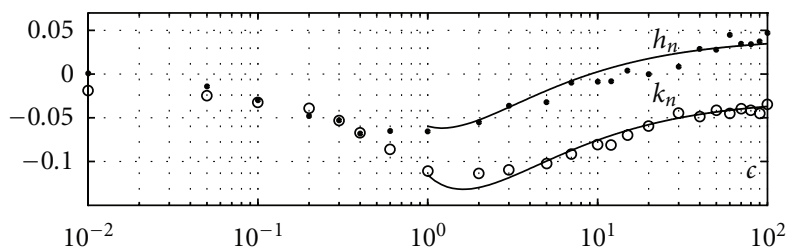

(b)

Figure 3: The points and circles are the numerical results of the variance $\sigma_{n}^{2}$ and the average number $\langle n\rangle$ versus $c$ for the values of the parameters chosen equal to $a=1, b=1, t=1$. The theoretical approximations calculated for $c \gg 1$ are given by (33a) and (33b) respectively (a) and drawn in solid lines. The points and circles are the numerical results of the differences $h_{n}=P(0)-P(1)$ and $k_{n}=Q(0)-Q(1)$ versus $c$ for $a=1, b=1, t=1$. The theoretical approximations calculated for $c \gg 1$ are given by (34a) and (34b) respectively (b) and drawn in solid lines.

As an application of these results to communications, we consider a system of communication processed with a direct threshold detector. The decision device operates such that

$$
\begin{gathered}
n \leqq n_{s}(c) \longrightarrow \mathrm{H}_{0} \longrightarrow P_{c=0}(n)=\mathrm{e}^{-a} \frac{a^{n}}{n !}, \\
n>n_{s}(c) \longrightarrow \mathrm{H}_{1} \longrightarrow P_{c \neq 0}(n),
\end{gathered}
$$

based on the binary hypotheses: $\mathrm{H}_{0}$ (no correlation) and $\mathrm{H}_{1}$ (correlation with the parameter $c$ ). The threshold is obtained from the likelihood ratio $\Lambda(n)$,

$$
\Lambda(n)=\frac{P_{c \neq 0}(n)}{P_{c=0}(n)}>1 \longrightarrow n_{s}(c) .
$$

We can also utilize the TIDs as a useful tool of processing [12]. The threshold would be based on the likelihood ratio $\Theta(t)=w_{c}(t) / \lambda \mathrm{e}^{-\lambda t}>1 \rightarrow t_{s}(c)$ and the decision would operate as follows

$$
\begin{gathered}
t \leq t_{s}(c) \longrightarrow \mathrm{H}_{1} \longrightarrow w_{c}(t)=K \frac{\mathrm{e}^{-a t}}{b+c t}, \\
t \geq t_{s}(c) \longrightarrow \mathrm{H}_{0} \longrightarrow a \mathrm{e}^{-a t},
\end{gathered}
$$

where $t_{s}(c)=(1 / \mathrm{c})(K / \mathrm{a}-b), K$ being given by $(11)$.

Here, we focus on the method based on PNDs because it is generally more efficient. To simplify the calculations, the decision is not randomized [13].

Now, the probability of error in detection when processing with the relaxed PND is given by

$$
P_{\text {err }}=\frac{1}{2}\left(1-\sum_{n=0}^{n_{s}} P_{c=0}(n)+\sum_{n=0}^{n_{s}} P_{c \neq 0}(n)\right)
$$

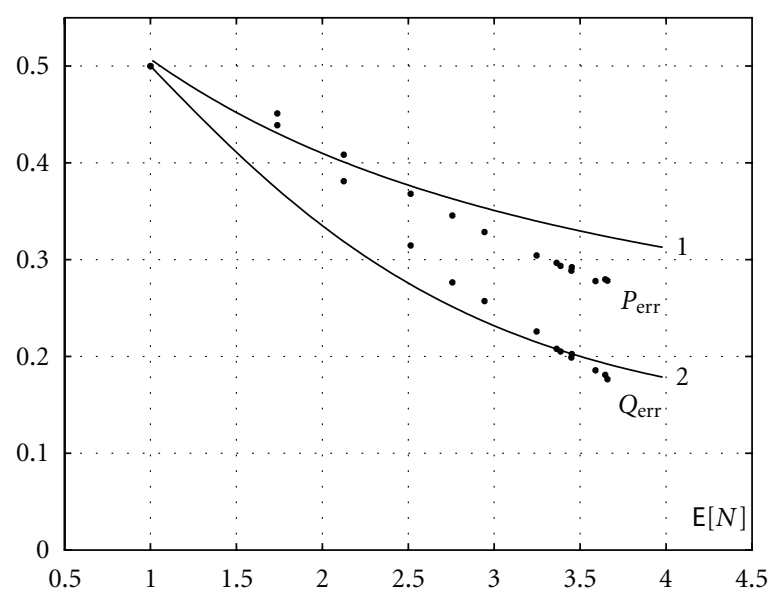

Figure 4: Plots of the probabilities of error $P_{\text {err }}$ and $Q_{\text {err }}$ versus the average number $E[N]$, respectively for the relaxed and the triggered processing. The values of the parameters are chosen equal to $a=$ $1, b=1, t=1$. The average number $\mathrm{E}[N](c)$ is the average number of the PDF $P_{c \neq 0}(n)$ for $0 \leq c \leq 100$, with $\mathrm{E}[N](0)=a$. The curves quoted " 1 " and " 2 " have been obtained from (41) and (42) respectively.

Similarly, we have for the processing with the triggered PND

$$
Q_{\text {err }}=\frac{1}{2}\left(1-\sum_{n=0}^{n_{s}} P_{c=0}(n)+\sum_{n=0}^{n_{s}} Q_{c \neq 0}(n)\right) .
$$

It is first seen that $Q_{\text {err }} \leq P_{\text {err }}$, the inequality which is demonstrated for a special case in the Appendix B. Furthermore, both probabilities of error in detection decrease with $\mathrm{E}[N]$, then with $c$ because $\mathrm{E}[N] \propto \log c$ as it is calculated in (28a), (33a) and is shown in Figure 3. This is within the range of $c: 0 \leq c \lesssim 100$. For instance, with the help of (30)-(31b) and (38)-(39) calculated for $n_{s}=1$ and $c=100$, we obtained $P_{\text {err }} \simeq 0.28$ and $Q_{\text {err }} \simeq 0.16$, the values which are in excellent agreement with the simulated results of Figure 4 .

On the other hand, the bounds to $Q_{\text {err }}$ and $P_{\text {err }}$ can easily be calculated. In fact, denoting

$$
v=\frac{\mathrm{E}[N]}{1+\mathrm{E}[N]},
$$

where $\mathrm{E}[N]=a+\xi$. Now, taking into account (35a), we have up to $O\left(\xi^{2}\right)$,

$$
\begin{gathered}
P_{1}(n)=(1-v) \nu^{n}, \\
P_{\mathrm{err}}^{(1)} \simeq 1-\frac{1}{e}-\frac{\nu^{2}}{2} \simeq 1-\frac{1}{e}-\frac{\xi}{8},
\end{gathered}
$$

for $0 \leq v \neq 1$. Therefore $P_{\text {err }}^{(1)}$ is the approximation of the exact $P_{\text {err }}$ for $n_{s}=1$ plotted as the curve quoted "1" in Figure 4 where $a=1$. Similarly, taking into account (35a), we have

$$
\begin{gathered}
P_{1}(n)=\mathrm{e}^{-\mathrm{E}[N]} \frac{\mathrm{E}[N]^{n}}{n !}, \\
P_{\text {err }}^{(2)} \simeq \frac{1}{2}-\frac{1}{e}+\frac{1+\mathrm{E}[N]}{2} \mathrm{e}^{-\mathrm{E}[N]} \simeq \frac{1}{2}-\frac{1}{e} \xi .
\end{gathered}
$$


Again, $P_{\mathrm{err}}^{(2)}$ is the approximation of the exact $P_{\mathrm{err}}$ for $n_{s}=1$ plotted as the the curve quoted " 2 " in Figure 4 for $a=1$. Finally, we have

$$
P_{\mathrm{err}}^{(2)} \leq Q_{\mathrm{err}} \leq P_{\mathrm{err}} \leq P_{\mathrm{err}}^{(1)}
$$

Let us conclude this analysis with a brief comment on information. We will concentrate on the cut-off rate which is known as a useful criterion for evaluating the performances of a channel. Thus, for a binary noiseless channel, when the transmission of messages " 0 " and " 1 " is done via the probabilities $P_{c=0}$ and $P_{c \neq 0}$ respectively (the probabilities $a$ priori are taken equal to $1 / 2$ ) the cut-off rate expresses as

$$
R=\log \frac{2}{1+\sum_{n=0}^{\infty} \sqrt{P_{c=0}(n) P_{c \neq 0}(n)}},
$$

which is generally interpreted as a lower bound to the channel capacity [14]. The $P_{c=0}(n)$ is the Poisson PND of parameter $a=1$ and $P_{c \neq 0}(n)$ will be, as above, either the relaxed or the triggered PND yielding the cut-off rates $R_{p}$ and $R_{q}$, respectively. Because exact closed expressions seem difficult to attain, the bounds are very useful and can easily be established. The first bound is obtained using geometric PNDs

$$
\begin{aligned}
P_{0}(n)=\left(1-\nu_{0}\right) \nu_{0}^{n}, \\
R_{1}=\log \frac{2}{1+\sqrt{\left(1-\nu_{0}\right)(1-\nu)} /\left(1-\sqrt{\nu_{0} \nu}\right)} \\
\simeq \underset{0 \leq \xi \lesssim 2}{\simeq} \frac{\xi^{2}}{32}\left(1-\frac{3 \xi}{4}+\frac{\xi^{2}}{2}\right),
\end{aligned}
$$

where $v$ is given by $(40)$ and $\nu_{0}=a /(1+a)$. This is a lower bound to $R_{p}$.

The second bound is obtained using the Poisson PNDs

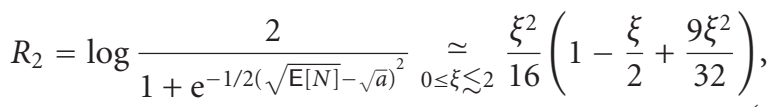

which is an upperbound to $R_{p}$.

In Figure 5, the results of the simulation $R_{p}$ and $R_{q}$ and the bounds (curves quoted " 1 " and " 2 ") given by ( $45 \mathrm{~b})$ and (46) are plotted versus $E[N]$. Here again, it is seen that the processing with the triggered PND performs much better that the processing with the relaxed $\mathrm{PND}$

$$
R_{1} \leq R_{p} \leq R_{2} \leq R_{q}
$$

in the limit of $0 \leq c \leq 100$. However, because the cut-off rate is a monotonic increasing function with respect to $\mathrm{E}[N]$, the inequalities (47) may be extrapolated to for all $c$.

In conclusion, the binary performances, as summarized by the inequalities (43) and (47), show that the processing with the triggered PND is the preferable mode of operation. Both performances, in detection and information transmission, are improved with the coefficient of correlation.

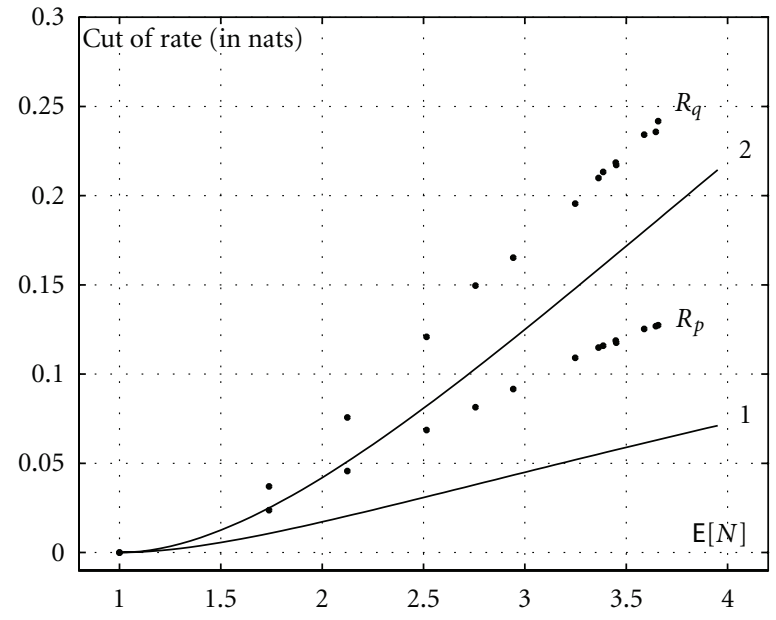

Figure 5: Plots of the cut-off rates $R_{p}$ and $R_{q}$ versus the average number $\mathrm{E}[N]$, respectively for the processing with the relaxed and the triggered PNDs. The values of the parameters are chosen equal to $a=1, b=1, t=1$. The average number $\mathrm{E}[N](c)$ is the average number of the PDF $P_{c \neq 0}(n)$ for $0 \leq c \leq 100, \mathrm{E}[N](0)=a$. The curves quoted " 1 " and " 2 " have been obtained from (45b) and (46) respectively.

\section{Appendices}

\section{A. Approximate Expressions}

A.1. The Exponential Integral Functions. The exponential integral functions can be expressed, up to $O\left(x^{3}\right)$,

$$
\begin{aligned}
& \operatorname{Ei}(1, x) \simeq-\gamma-\log x+x-\frac{x^{2}}{4}, \\
& \operatorname{Ei}(2, x) \simeq 1+(\gamma+\log x-1) x-\frac{x^{2}}{2}, \\
& \operatorname{Ei}(3, x) \simeq \frac{1}{2}-x-\left(\frac{1}{2} \gamma+\frac{1}{2} \log x-\frac{3}{4}\right) x^{2},
\end{aligned}
$$

where $\gamma$ is the Euler constant. For $x \rightarrow \infty$

$$
\begin{aligned}
& \operatorname{Ei}(1, x) \simeq \frac{\mathrm{e}^{-x}}{x}\left(1-\frac{1}{x}\right), \\
& \operatorname{Ei}(2, x) \simeq \frac{\mathrm{e}^{-x}}{x}\left(1-\frac{2}{x}\right) \\
& \operatorname{Ei}(3, x) \simeq \frac{\mathrm{e}^{-x}}{x}\left(1-\frac{3}{x}\right) .
\end{aligned}
$$

A.2. Probability of Number Distribution. Depending on the values of $c$, we have the following approximations of the first values of $P(n)$ :

$$
\begin{aligned}
& P(0) \simeq \mathrm{e}^{-a}\left(1-\omega-\frac{1}{a} \omega^{2}\right) \\
& P(1) \simeq \mathrm{e}^{-a}\left(a+(1-a) \omega+\left(\frac{1}{a}+\frac{a}{6}-\frac{3}{2}\right) \omega^{2}\right), \\
& P(2) \simeq \mathrm{e}^{-a}\left(\frac{a^{2}}{2}+a\left(1-\frac{a}{2}\right) \omega+\left(\frac{a^{2}}{8}+\frac{3}{2}-2 a\right) \omega^{2}\right),
\end{aligned}
$$


for $c \ll 1$ and where $\omega=c / b$. Similarly, it can be shown that

$$
\begin{aligned}
& P(0) \simeq 1+\frac{\gamma-\log c}{c}, \\
& P(1) \simeq \frac{\log c-\gamma}{c}, \\
& P(2) \simeq \frac{\gamma^{2}-2(1+\gamma) \log c}{c^{2}},
\end{aligned}
$$

for $c \gg 1$.

\section{B. Inequality between Probabilities of Error in Binary Detection}

To demonstrate that the triggered processing yields better performance than the relaxed processing, $\Delta_{e}=Q_{\mathrm{err}}-P_{\mathrm{err}} \leq$ 0 , we begin with (8)

$$
\begin{gathered}
P(n ; t)=\frac{1}{n !} \mathrm{E}\left[\mathcal{g}(t)^{n} \mathrm{e}^{-\mathscr{g}(t)}\right], \\
Q(n ; t)=\frac{1}{\mathrm{E}[N]} \frac{1}{n !} \mathrm{E}\left[\mathcal{g}(t)^{n+1} \mathrm{e}^{-\mathcal{g}(t)}\right],
\end{gathered}
$$

and show that for

$$
\begin{aligned}
& P_{\mathrm{err}}=\frac{1}{2}\left(1-\sum_{n=0}^{n_{s}} P_{c=0}(n)+\sum_{n=0}^{n_{s}} P_{c \neq 0}(n)\right), \\
& Q_{\mathrm{err}}=\frac{1}{2}\left(1-\sum_{n=0}^{n_{s}} P_{c=0}(n)+\sum_{n=0}^{n_{s}} Q_{c \neq 0}(n)\right),
\end{aligned}
$$

we have

$$
\begin{aligned}
& \Delta_{e}=Q_{\mathrm{err}}-P_{\mathrm{err}}, \\
& =\frac{1}{2}\left(\sum_{n=0}^{n_{s}} \mathrm{E}\left[\mathrm{e}^{-\mathcal{g}}\left(\frac{\mathscr{g}^{n+1}}{\mathrm{E}[\mathcal{g}]}-\mathscr{g}^{n}\right)\right]\right) \\
& =\frac{1}{2} \mathrm{E}\left[\sum_{n=0}^{n_{s}} \mathrm{e}^{-g}\left(\frac{\boldsymbol{g}^{n+1}}{\mathrm{E}[\boldsymbol{g}]}-\boldsymbol{g}^{n}\right)\right] \\
& \leq \frac{1}{2} \mathrm{E}\left[\sum_{n=0}^{n_{s}}\left(\frac{\mathscr{g}^{n+1}}{\mathrm{E}[\mathcal{g}]}-\mathscr{g}^{n}\right)\right]=\frac{1}{2} \sum_{n=0}^{n_{s}}\left(\frac{\mathrm{E}\left[\mathscr{g}^{n+1}\right]}{\mathrm{E}[\mathcal{g}]}-\mathrm{E}\left[\mathscr{g}^{n}\right]\right) .
\end{aligned}
$$

where we used $\mathrm{e}^{-\mathscr{g}} \leq 1, \boldsymbol{g} \geq 0$.

For $n_{s}=1$, noticing that $\mathrm{E}\left[\mathcal{g}^{2}\right] \geq \mathrm{E}[\mathrm{g}]^{2}$, we have $\Delta_{e} \leq 0$.

For higher values of $n_{s}$, this method seems not useful because it requires to prove that $E\left[g^{n+1}\right] / E[g] \geq$ $\mathrm{E}\left[g^{n}\right]$, for all $n$, which is not so easy although the inequality is true for several types of density distributions of interest in statistical optics.

\section{Disclosure}

Laboratoire des Signaux et Systèmes is a joint laboratory (UMR 8506) of CNRS. and École Supérieure d'Électricité is and associated with the Université Paris-Orsay, France.

\section{References}

[1] C. Bendjaballah, "Nonclassical second-order photon statistics," Journal of Physics B, vol. 39, no. 4, pp. 783-803, 2006.

[2] C. Bendjaballah, "Nonclassical photon statistics," Physical Review A, vol. 73, no. 5, Article ID 053816, 2006.

[3] C. Bendjaballah, Introduction to Photon Communication, Springer, Heidelberg, Germany, 1995.

[4] J. A. McFadden, "On the lengths of intervals in a stationary point process," Journal of the Royal Statistical Society B, vol. 24, pp. 364-382, 1962.

[5] C. Godrèche and J. M. Luck, "Statistics of the occupation time of renewal processes," Journal of Statistical Physics, vol. 104, no. 3-4, pp. 489-524, 2001.

[6] C. Bendjaballah, "Statistical properties of several models of fractional random point processes," Communications in Nonlinear Science and Numerical Simulation, vol. 16, pp. 32243236, 2011.

[7] B. C. Arnold and D. Strauss, "Bivariate distributions with exponential conditionals," Journal of the American Statistical Association, vol. 83, pp. 522-527, 1988.

[8] S. Nadarajah and S. Kotz, "Reliability for some bivariate exponential distributions," Mathematical Problems in Engineering, vol. 2006, pp. 1-14, 2006.

[9] I. S. Gradshteyn and I. M. Ryzhik, Table of Integrals, Series, and Products, Academic Press, New York, NY, USA, 6th edition, 2000.

[10] B. Picinbono and C. Bendjaballah, "Characterization of nonclassical optical fields by photodetection statistics," Physical Review A, vol. 71, no. 1, Article ID 013812, 12 pages, 2005.

[11] Y. Yu, "Efficient simulation of a bivariate exponential conditionals distribution," Computational Statistics and Data Analysis, vol. 52, no. 5, pp. 2273-2276, 2008.

[12] C. Bendjaballah and K. Hassan, "Probability of detecting a coherent optical signal in thermal noise," Journal of the Optical Society of America A, vol. 73, pp. 1840-1843, 1983.

[13] C. W. Helstrom, Quantum Detection and Estimation Theory, Academic Press, New York, NY, USA, 1976.

[14] J. L. Massey, "Capacity, cut-off rate and coding for a directdetection optical channel," IEEE Transactions on Communications, vol. 29, no. 11, pp. 1615-1621, 1981. 

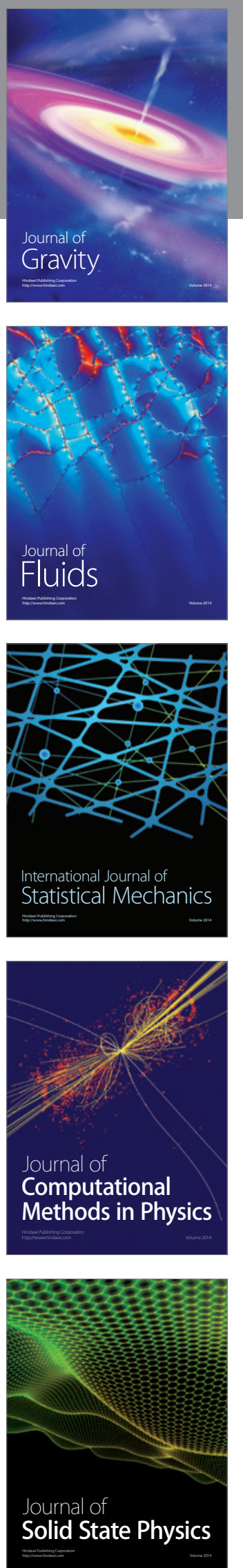
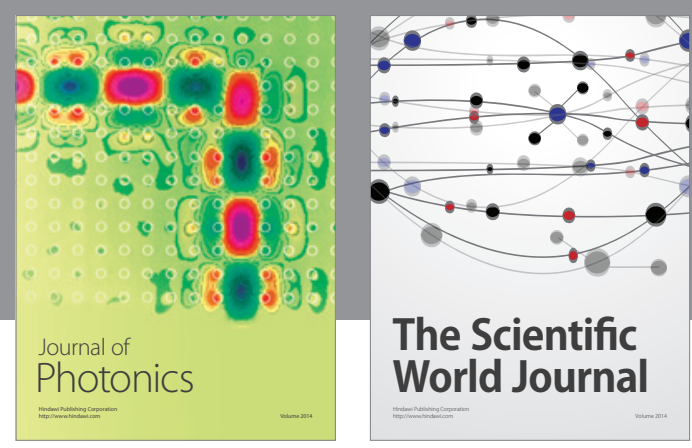

The Scientific World Journal

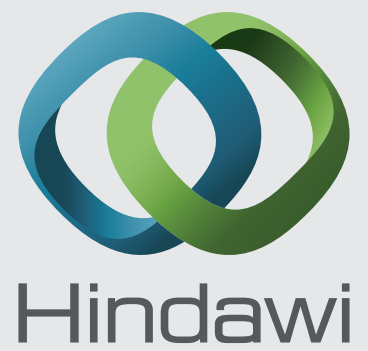

Submit your manuscripts at http://www.hindawi.com
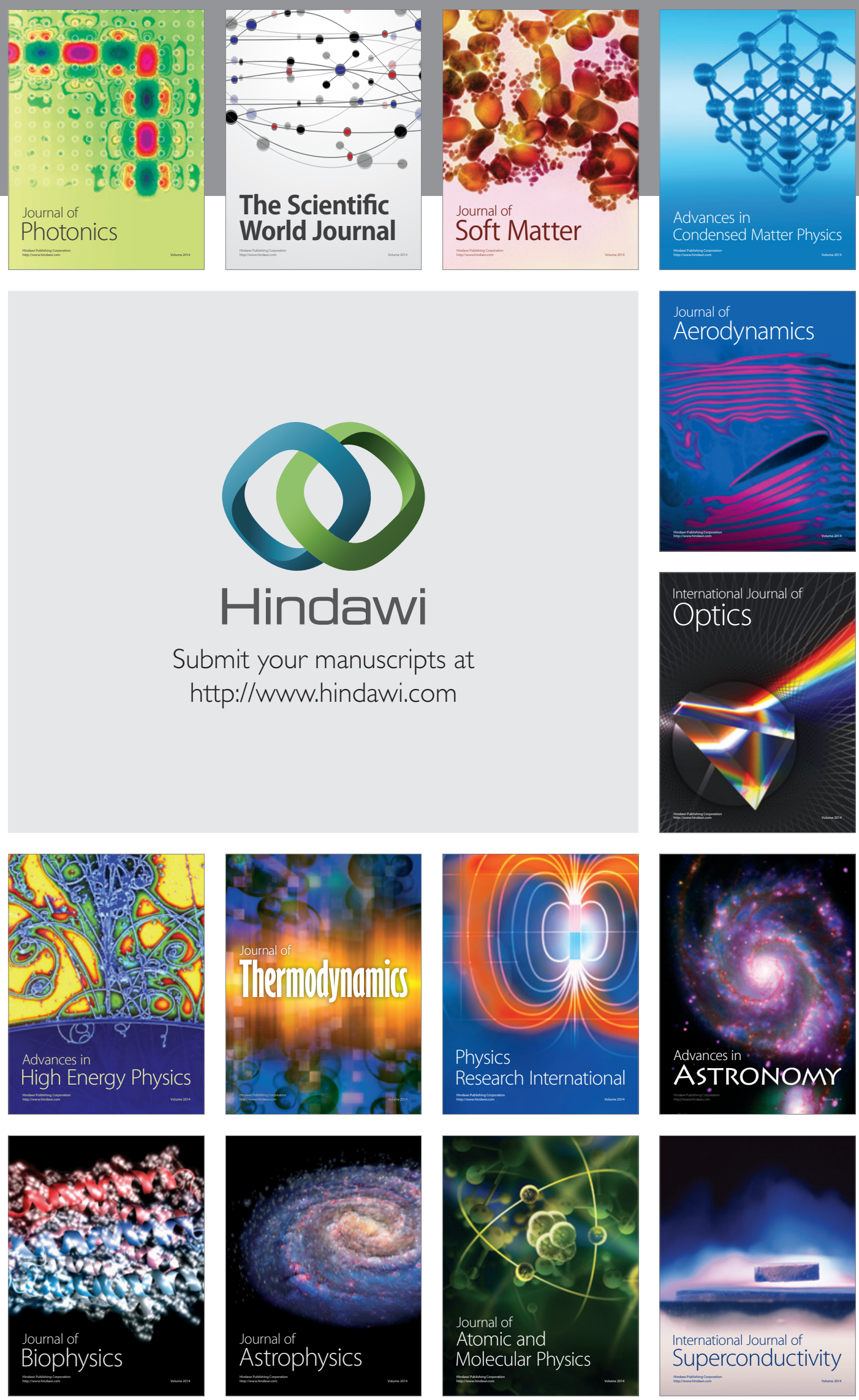
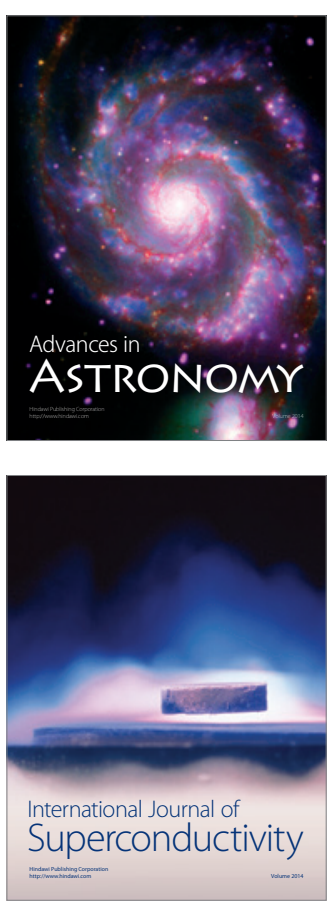